\title{
Robust fault-tolerant control for flexible spacecraft against partial actuator failures
}

\author{
Ran Zhang • Jianzhong Qiao • Tao Li · Lei Guo
}

Received: 23 July 2013 / Accepted: 4 January 2014 / Published online: 30 January 2014

(C) The Author(s) 2014. This article is published with open access at Springerlink.com

\begin{abstract}
In this paper, we present a robust faulttolerant control scheme to achieve attitude control of flexible spacecraft with disturbances and actuator failures. It is shown that the control algorithms are not only attenuate exogenous bounded disturbances with attenuation level, but also able to tolerate partial loss of actuator effectiveness. The proposed controller design is simple and can guarantee the faulty closed-loop system to be quadratically stable with a prescribed upper bound of the cost function. The design algorithms are obtained by combining free weighting matrices method with linear matrix inequality technique. The effectiveness of the proposed design method is demonstrated in a spacecraft attitude control system subject to loss of actuator effectiveness.
\end{abstract}

Keywords Flexible spacecraft · Fault tolerant . $H_{\infty}$ control $\cdot$ LMI

\section{Introduction}

High precision attitude control has been a difficult and important problem for flexible spacecraft in commu-

R. Zhang $\cdot$ J. Qiao $\cdot$ L. Guo

School of Instrumentation Science and Opto-Electronics Engineering, Beihang University, Beijing 100191, China

T. Li (凶)

Institute of Advanced Technology, Nanjing University of Posts and Telecommunications, Nanjing 210046, China e-mail: litaojia@163.com nication, navigation, remote sensing, and other spacerelated missions. It is because modern spacecraft often employ large, deployed and light damping structures (such as solar paddles and antenna reflectors) to provide sufficient power supply and reduce launch costs [1-6]. During the control of the rigid body attitude, actuators play an important role of linking control commands to physical actions $[7,8]$. Normally, the actuators should execute commands demanded by the controller faithfully and completely. In this condition, the actuators need to be $100 \%$ effective. However, when a fault occurs in the actuator, the handicapped actuator may not complete the control command fully. Naturally, the control channel effectiveness (or lack of it) becomes an appropriate measure of the severity of the actuator fault [9]. In an spacecraft, actuator faults may cause discrepancies between the desired and the actual movements of these control surfaces due to incorrect supply pressure in the hydraulic lines, change in hydraulic compliance, and line leakage [10]. Any of these problems can prevent the primary control surfaces such as elevators, ailerons, or rudder from moving to the positions demanded by the controller [9]. On the other hand, the complex space structure may lead to the decreased rigidity and low-frequency elastic modes. However, elastic vibration of the flexible appendages may cause degradation of the performance of attitude control $[7,11]$. Thus, the desired control scheme should tolerate partial loss of actuator effectiveness and be robust enough to overcome various disturbances from structural vibrations of the flexible appendages. 
Due to the increasing demands for high reliability and survivability of the complex control systems, the fault-tolerant control (FTC) has attracted extensive interests and attention [12-21]. FTC can be divided into passive FTC $[12,13]$ and active ones $[14,15]$. An active FTC uses the diagnosis results provided by the fault detection and diagnosis to actively adjust the control efforts, thus is potentially capable of dealing with a larger number of faults $[14,15]$. Compared with the active FTC, the passive one has the advantage of not requiring the exact actuator fault information; thus, it is simple to implement. The passive FTC can also ensure system stability and desired performance after the actuator fault occurs and before the fault detection and diagnosis phase finishes [12,13].

Motivated by the preceding discussion, in this paper, a passive FTC scheme for flexible spacecraft with disturbances and partial loss of actuator effectiveness is studied. First, the partial loss of actuator effectiveness problem is transformed into uncertain parameters problem. Second, the fault tolerant control is designed by combining $H_{\infty}$ control technique and robust control method. The proposed control algorithms are not only attenuate disturbances from structural vibrations of the flexible appendages with $H_{\infty}$ attenuation level, but also able to robust to partial loss of actuator effectiveness. Meanwhile, the resultant FT controller may be simply designed and can guarantee the faulty closed-loop system to be quadratically stable with a prescribed upper bound of the cost function. Finally, a numerical example is shown to demonstrate the good performance of our method.

The rest of this paper is organized as follows. The single-axis model of flexible spacecraft model and partial loss of actuator effectiveness are described in Sect. 2. The passive FT controller is designed and analyzed in Sect. 3. Numerical simulations on different control effectiveness factor situations are presented in Sect. 4 to demonstrate the performance of the proposed control method. Finally, we conclude the paper in Sect. 5.

Notation: Throughout this paper, $R^{n}$ denotes the $n$ dimensional Euclidean space; the space of squareintegrable vector functions over $[0, \infty)$ is denoted by $l_{2}[0, \infty)$; the superscripts " $T$ " and " -1 " stand for matrix transposition and matrix inverse, respectively; $P>(\geq 0)$ means that $P$ is real symmetric and positive definite (semidefinite). The identity andzero matrices are denoted by $I$ and 0 , respectively, with appropriate dimensions. In symmetric block matrices or complex matrix expressions, $\operatorname{diag}\{\ldots\}$ stands for a blockdiagonal matrix, and $*$ represents a term that is induced by symmetry. For a vector $v(t)$, its norm is given by $\|v(t)\|_{2}=\int_{0}^{\infty} v^{\top}(t) v(t) \mathrm{d} t$. Matrices, if their dimensions are not explicitly stated, are assumed to be compatible for related algebraic operations.

\section{Problem formulation and preliminaries}

Similarly to the references $[11,22]$, the single-axis model can be derived from the nonlinear attitude dynamics of the flexible spacecraft. In this paper, the problem is simplified and only considers the singleaxis rotational maneuver. It is assumed that this model includes one rigid body and one flexible appendage, and the relative elastic spacecraft model is described as:

$$
\left\{\begin{array}{l}
J \ddot{\theta}(t)+F \ddot{\eta}(t)=u^{F}(t) \\
\ddot{\eta}(t)+C_{\mathrm{m}} \dot{\eta}(t)+\Lambda \eta(t)+F^{\top} \ddot{\theta}(t)=0,
\end{array}\right.
$$

where $\theta(t)$ is the attitude angle, $J$ is the spacecraft inertia about the pitch axis, $\eta(t)$ is the flexible modal coordinate, $F$ is the rigid-elastic coupling matrix, $u^{F}(t)$ is the control torque generated by the reaction wheels that are installed in the flexible spacecraft. $C_{\mathrm{m}}=\operatorname{diag}\left\{2 \xi_{1} \varpi_{1}, \ldots 2 \xi_{n} \varpi_{n}\right\}$ is modal damping matrix, where $\xi_{i},(i=1, \ldots n)$ is the damping ratio, and $\varpi_{i},(i=1, \ldots n)$ is the modal frequency. $\Lambda=\operatorname{diag}\left\{\varpi_{1}^{2}, \ldots \varpi_{n}^{2}\right\}$ is stiffness matrix. $n$ is their dimensions. Since vibration energy is concentrated in low-frequency modes in a flexible structure, its reduced order model can be obtained by modal truncation. In this paper, only the first two bending modes are taken into account. Then we can get

$$
\left(J-F F^{\top}\right) \ddot{\theta}=F\left(C_{\mathrm{m}} \dot{\eta}(t)+\Lambda \eta(t)\right)+u^{F}(t) .
$$

To formulate the FTC problem, the fault model must be established. According to the fault type for flightcontrol system established in $[15,18]$, the fault type considered in this study is the loss of actuator effectiveness. We use $u^{F}(t)$ to describe the control signal as follows:

$u^{F}(t)=\omega u(t)$,

where $\omega$ is the control effectiveness factor and satisfied by the following form: 


$$
\begin{gathered}
\omega=\operatorname{diag}\left\{\omega_{1}, \omega_{2}, \ldots, \omega_{n}\right\}, \omega_{i} \in\left[\underline{\omega}_{i} \bar{\omega}_{i}\right] \\
i=1,2 \ldots, n, 0 \leq \underline{\omega}_{i} \leq \bar{\omega}_{i} \leq 1,
\end{gathered}
$$

where $\omega_{i}$ is an unknown constant, $\underline{\omega}_{i}$ and $\bar{\omega}_{i}$ represent the known lower and upper bounds of $\omega_{i}$, respectively. For simplicity, we introduce the following notation:

$$
\begin{gathered}
\hat{\omega}=\operatorname{diag}\left\{\hat{\omega}_{1}, \hat{\omega}_{2}, \ldots, \hat{\omega}_{n}\right\}, \\
J=\operatorname{diag}\left\{j_{1}, j_{2}, \ldots, j_{n}\right\}, \\
L=\operatorname{diag}\left\{l_{1}, l_{2}, \ldots, l_{n}\right\},
\end{gathered}
$$

where

$$
\begin{gathered}
\hat{\omega}_{i}=\frac{1}{2}\left(\underline{\omega}_{i}+\bar{\omega}_{i}\right), \quad j i=\frac{\bar{\omega}_{i}-\underline{\omega}_{i}}{\bar{\omega}_{i}+\underline{\omega}_{i}}, \\
l_{i}=\frac{\omega_{i}-\hat{\omega}_{i}}{\hat{\omega}_{i}}, i=1,2, \ldots, n .
\end{gathered}
$$

Then, we have

$$
\begin{aligned}
& \omega=\hat{\omega}(I+L),|L| \leq J \leq I, \\
& |L|=\operatorname{diag}\left\{\left|l_{1}\right|,\left|l_{2}\right|, \ldots,\left|l_{n}\right|\right\} .
\end{aligned}
$$

Remark 1 When $\underline{\omega}_{i}=\bar{\omega}_{i}=1$, then the $i$ th actuator is considered to be fault-free. Nevertheless, when $0 \leq \omega_{i}<1$, the considered fault is a partial loss of control effectiveness. Specially, when $\omega_{i}=0$, the $i$ th actuator is considered to be failure and the actuator is out of order. On the other hand, it is noted that loss of actuator effectiveness problem may be transformed into uncertain parameters problem by using fault description method in (7), which will also make the partial actuator failures problem to easily solve in subsequent section.

Letting $x(t)=\left[\begin{array}{ll}\theta^{\top}(t) & \dot{\theta}^{\top}(t)\end{array}\right]^{\top}$, then, the system (2) with actuator faults (7), can be transformed into the state-space form

$$
\left\{\begin{array}{l}
\dot{x}(t)=A x(t)+B \omega u(t)+B w(t) \\
y(t)=C x(t)
\end{array}\right.
$$

where $y(t)$ is measured reference output, $w(t)=$ $F\left(C_{\mathrm{m}} \dot{\eta}(t)+\Lambda \eta(t)\right)$ is as the disturbance due to elastic vibration of the flexible appendages, which is supposed $w(t)$ belongs to $l_{2}[0, \infty)$ and satisfied $\|w(t)\| \leq \delta$ and

$$
A=\left[\begin{array}{ll}
0 & I \\
0 & 0
\end{array}\right], B=\left[\begin{array}{c}
0 \\
\left(J-F F^{\top}\right)^{-1}
\end{array}\right], C=I .
$$

For system (8), the following FT controller is employed via state feedback

$u(t)=K x(t)$, where $K$ is the gain of controller and need to design. Then, with the control law (9), the system (8) can be expressed as follows

$\left\{\begin{array}{l}\dot{x}(t)=(A+B \omega K) x(t)+B w(t) \\ y(t)=C x(t) .\end{array}\right.$

The objective of this paper is to design FT controller such that

- The closed-loop system (10) with $w(t)=0$ is asymptotically stable;

- In the case when $w(t)=0$, the following cost function associated with closed-loop system (10) satisfies

$$
J_{c}=\int_{0}^{\infty} x^{\top}(t) Z x(t)+u^{\top}(t) \omega^{\top} R \omega u(t) \mathrm{d} t \leq J^{*},
$$

where $J^{*}>0$ is a specified constant, $Z$ and $R$ are given positive definite matrices;

- Under the zero initial condition, the closed-loop system (10) satisfies $\|y\|_{2}<\gamma\|w(t)\|$, for any nonzero $w(t)$ that belongs to $l_{2}[0,1)$, where $\gamma>0$ is a prescribed scalar.

Now, we give the following lemma which are needed in the proof of main results.

Lemma 1 ([23]) Given matrices $M=M^{\top}, S$ and $N$ of appropriate dimensions, the inequality

$M+S \Delta(t) N+N^{\top} \Delta^{\top}(t) S^{\top}<0$

holds for all $\Delta(t)$ such that $\Delta^{\top}(t) \Delta(t) \leq I$, if and only if, there exists $\delta>0$

$\left[\begin{array}{ccc}M & \delta S & N^{\top} \\ \delta S^{\top} & -\delta I & 0 \\ N & 0 & -\delta I\end{array}\right]<0$.

Lemma 2 (Schur complement [23]) Given constant matrices $\Omega_{1}, \Omega_{2}, \Omega_{3}$ where $\Omega_{1}=\Omega_{1}^{\top}$ and $\Omega_{2}>0$, then

$\Omega_{1}+\Omega_{3}^{\top} \Omega_{2}^{-1} \Omega_{3}<0$

if only if

$\left[\begin{array}{cc}\Omega_{1} & \Omega_{3}^{\top} \\ \Omega_{3} & -\Omega_{2}\end{array}\right]<0$, or $\left[\begin{array}{cc}-\Omega_{2} & \Omega_{3} \\ \Omega_{3}^{\top} & \Omega_{1}\end{array}\right]<0$. 


\section{Fault-tolerant control design}

In this section, we will give design method based on LMI to compute the FT controller gain.

\subsection{Stability of closed-loop system}

Theorem 1 Given a scalar $\gamma>0$ and matrices $Z>$ $0, R>0$, the system (10) is asymptotically stable and satisfies $\|y(t)\|_{2}<\gamma\|w(t)\|_{2}$ for any non-zero $w(t) \in l_{2}[0, \infty)$ under the zero initial condition if there exist a matrix $P>0$, invertible matrices $\Gamma_{1}$ and $\Gamma_{2}$ such that the following inequality holds:
Proof The first step is to analyze the asymptotic stability of system (10). Consider system (10) in the absence of $w(t)$, we choose a Lyapunov function as $V(t)=x^{\top}(t) P x(t)$. For any matrix $\Gamma_{1}$ and invertible matrix $\Gamma_{2}$ of appropriate dimensions, we have

$$
\begin{aligned}
& {\left[x^{\top}(t) \Gamma_{1}^{\top}+\dot{x}^{\top}(t) \Gamma_{2}^{\top}\right]} \\
& \quad \times[-\dot{x}(t)+(A+B \omega K) x(t)]=0 .
\end{aligned}
$$

Differentiating $V(t)$ along the trajectory of the system (10) and adding (14) to it gives

$$
\begin{aligned}
& \dot{V}(t)+\left[x^{\top}(t) \Gamma_{1}^{\top}+\dot{x}^{\top}(t) \Gamma_{2}^{\top}\right][-\dot{x}(t)+(A+B \omega K) x(t)] \\
& =2 x^{\top}(t) P \dot{x}(t)+2\left[x^{\top}(t) \Gamma_{1}^{\top}+\dot{x}^{\top}(t) \Gamma_{2}^{\top}\right][-\dot{x}(t)+(A+B \omega K) x(t)] \\
& =2 x^{\top}(t) P \dot{x}(t)-2 x^{\top}(t) \Gamma_{1}^{\top} \dot{x}(t)+2 x^{\top}(t) \Gamma_{1}^{\top}(A+B \omega K) x(t)-2 \dot{x}^{\top}(t) \Gamma_{2}^{\top} \dot{x}(t)+2 \dot{x}^{\top}(t) \Gamma_{2}^{\top}(A+B \omega K) x(t) \\
& =\left[\begin{array}{ll}
x^{\top}(t) & \dot{x}^{\top}(t)
\end{array}\right]\left[\begin{array}{cc}
(A+B \omega K)^{\top} \Gamma_{1}+\Gamma_{1}^{\top}(A+B \omega K) & P-\Gamma_{1}^{\top}+(A+B \omega K)^{\top} \Gamma_{2} \\
* & -\Gamma_{2}-\Gamma_{2}^{\top}
\end{array}\right]\left[\begin{array}{c}
x(t) \\
\dot{x}(t)
\end{array}\right]
\end{aligned}
$$

$$
\left[\begin{array}{cccc}
(A+B \omega K)^{\top} \Gamma_{1}+\Gamma_{1}^{\top}(A+B \omega K)+Z+K^{\top} \omega^{\top} R \omega K & P-\Gamma_{1}^{\top}+(A+B \omega K)^{\top} \Gamma_{2} & \Gamma_{1}^{\top} B & C^{\top} \\
* & -\Gamma_{2}-\Gamma_{2}^{\top} & \Gamma_{2}^{\top} B & 0 \\
* & * & -\gamma^{2} I & 0 \\
* & * & * & -I
\end{array}\right]<0 .
$$

Furthermore, an upper bound of performance index (11) is given by

$J_{c} \leq x^{\top}(0) P x(0)$.
According to (12) and Lemma 2, it implies $\dot{V}(t)<$ 0 . Hence, the system (10) is asymptotically stable. Next, we consider the upper bound of cost function (11). It is noted that

$$
\begin{aligned}
& \dot{V}(t)+x^{\top}(t) Z x(t)+u^{\top}(t) \omega^{\top} R \omega u(t) \\
& =2 x^{\top}(t) P \dot{x}(t)+x^{\top}(t) Z x(t)+x^{\top}(t) K^{\top} \omega^{\top} R \omega K x(t)+2\left[x^{\top}(t) \Gamma_{1}^{\top}+\dot{x}^{\top}(t) \Gamma_{2}^{\top}\right][-\dot{x}(t)+(A+B \omega K) x(t)] \\
& =\left[x^{\top}(t) \dot{x}^{\top}(t)\right]\left[\begin{array}{cc}
(A+B \omega K)^{\top} \Gamma_{1}+\Gamma_{1}^{\top}(A+B \omega K)+Z+K^{\top} \omega^{\top} R \omega K & P-\Gamma_{1}^{\top}+(A+B \omega K)^{\top} \Gamma_{2} \\
* & -\Gamma_{2}-\Gamma_{2}^{\top}
\end{array}\right]\left[\begin{array}{c}
x(t) \\
\dot{x}(t)
\end{array}\right]
\end{aligned}
$$


When (12) holds, we have

$$
\dot{V}(t)+x^{\top}(t) Z x(t)+u^{\top}(t) \omega^{\top} R \omega u(t)<0 .
$$

Integrating both sides of (17) from $t=0$ to $t=\infty$, we obtain

$$
\begin{aligned}
J_{c}= & \int_{0}^{\infty} x^{\top}(t) Z x(t)+u^{\top}(t) \omega^{\top} R \omega u(t) \mathrm{d} t \\
& \leq-\int_{0}^{\infty} \dot{V}(t) \mathrm{d} t \leq x^{\top}(0) P x(0) .
\end{aligned}
$$

At last, we shall establish the $H_{\infty}$ performance of the system (10) under zero initial condition. Let

$J_{h}=\int_{0}^{t}\left[y^{\top}(s) y(s)-\gamma^{2} w^{\top}(s) w(s)\right] \mathrm{d} s$.

It can be shown that for any non-zero $w(t) \in l_{2}[0, \infty)$ and $t>0$,

$J_{h} \leq \int_{0}^{t}\left[y^{\top}(s) y(s)-\gamma^{2} w^{\top}(s) w(s)+\dot{V}(s)\right] \mathrm{d} s$.

It is clear that for any matrix $\Gamma_{1}$ and invertible matrix $\Gamma_{2}$ of appropriate dimensions, the following equality always is true

$$
\begin{aligned}
& {\left[x^{\top}(t) \Gamma_{1}^{\top}+\dot{x}^{\top}(t) \Gamma_{2}^{\top}\right]} \\
& \quad \times[-\dot{x}(t)+(A+B \omega K) x(t)+B w(t)]=0 .
\end{aligned}
$$

invertible matrices rather than positive definite matrices. Meanwhile, it is noted that $\Gamma_{1}$ and $\Gamma_{2}$ are not invertible matrices, equality (14) also is true. Here, in order to design fault tolerant controller gain, we assume them to invertible matrices.

\subsection{Controller design}

On the basis of Theorem 1, we will present a design method of robust FT controller in the following.

Theorem 2 For given scalars $\gamma>0, \kappa$ and matrices $Z>0, R>0$, if there exist a scalar $\delta>0$, a matrix $\hat{P}>0$, invertible matrix $V_{1}$ and any matrix $W$ such that the following inequality holds:

$$
\left[\begin{array}{cccccccc}
\Xi_{11} & \Xi_{12} & B & V_{1}^{\top} C^{\top} & V_{1}^{\top} & W^{\top} \hat{\omega} & \delta B \hat{\omega} & W^{\top} \\
* & \Xi_{22} & \kappa B & 0 & 0 & 0 & \delta \kappa B \hat{\omega} & 0 \\
* & * & -\gamma^{2} I & 0 & 0 & 0 & 0 & 0 \\
* & * & * & -I & 0 & 0 & 0 & 0 \\
* & * & * & * & -Z^{-1} & 0 & 0 & 0 \\
* & * & * & * & * & -R^{-1} & \delta \hat{\omega} & 0 \\
* & * & * & * & * & * & -\delta I & 0 \\
* & * & * & * & * & * & * & -\delta I
\end{array}\right]<0
$$

the closed-loop system (10) is asymptotically stable under the FT controller $u(t)=W V_{1}^{-1} x(t)$ and satisfies $\|y(t)\|_{2}<\gamma \quad\|w(t)\|_{2}$ for any non-zero $w(t) \in l_{2}[0, \infty)$ with the zero initial condition. Moreover, an upper bound of performance index (11) is given by

Then, we have

$$
\begin{aligned}
& y^{\top}(s) y(s)-\gamma^{2} w^{\top}(s) w(s)+\dot{V}(s)=\left[x^{\top}(t) \dot{x}^{\top}(t) w^{\top}(t)\right] \\
& \quad \times\left[\begin{array}{ccc}
C^{\top} C+(A+B \omega K)^{\top} \Gamma_{1}+\Gamma_{1}^{\top}(A+B \omega K) & P-\Gamma_{1}^{\top}+(A+B \omega K)^{\top} \Gamma_{2} & \Gamma_{1}^{\top} B \\
* & -\Gamma_{2}-\Gamma_{2}^{\top} & \Gamma_{2}^{\top} B \\
* & * & -\gamma^{2} I
\end{array}\right]\left[\begin{array}{c}
x(t) \\
\dot{x}(t) \\
w(t)
\end{array}\right]
\end{aligned}
$$

According to (21) and (20), $J_{h}<0$ follows from (12), which implies that $\|y(t)\|_{2}<\gamma\|w(t)\|_{2}$ holds for any non-zero $w(t) \in l_{2}[0, \infty)$. This complete the proof.

Remark 2 In Theorem 1, slack variables $\Gamma_{1}$ and $\Gamma_{2}$ are introduced. It is noted that $\Gamma_{1}$ and $\Gamma_{2}$ are useless for reducing the conservatism of stability conditions in $[24,25]$. However, they can more relaxed design of fault tolerant controller later on since they need only be
$J_{c} \leq x^{\top}(0)\left(V_{1}^{-\top} \hat{P} V_{1}^{-1}\right) x(0)$

where

$\Xi_{11}=V_{1}^{\top} A^{\top}+A V_{1}+W^{\top} \hat{\omega} B^{\top}+B \hat{\omega} W$

$\Xi_{12}=\hat{P}-V_{1}+\kappa V_{1}^{\top} A^{\top}+\kappa W^{\top} \hat{\omega} B^{\top}$

$\Xi_{22}=-\kappa V_{1}-\kappa V_{1}^{\top}$.

Proof Suppose there exists $\delta>0$ such that the inequality (22) holds and Let 
$M=\left[\begin{array}{cccccc}\Xi_{11} & \Xi_{12} & B & V_{1}^{\top} C^{\top} & V_{1}^{\top} & W^{\top} \hat{\omega} \\ * & \Xi_{22} & \kappa B & 0 & 0 & 0 \\ * & * & -\gamma^{2} I & 0 & 0 & 0 \\ * & * & * & -I & 0 & 0 \\ * & * & * & * & -Z^{-1} & 0 \\ * & * & * & * & * & -R^{-1}\end{array}\right]$

$$
S=\left[\begin{array}{c}
B \hat{\omega} \\
\kappa B \hat{\omega} \\
0 \\
0 \\
0 \\
\hat{\omega}
\end{array}\right], N=\left[\begin{array}{lllll}
W & 0 & 0 & 0 & 0
\end{array}\right]
$$

From (7), we can obtain $L^{\top} L \leq I$. According to Lemma 1 , there are $M+S L N+N^{\top} L^{\top}(t) S^{\top}<0$ holds, that is
Theorem 2, which only depends on the control effectiveness factor $\hat{\omega}$. On the other hand, the designed controller is described in form of LMI, which is easy to solve by using Matlab LMI toolbox.

\section{Numerical examples}

In this section, the faut tolerant control scheme will be applied to a spacecraft with one flexible appendage. Since low-frequency modes are generally dominant in a flexible system, only the lowest two bending modes have been considered for the implemented spacecraft model. Thus, we suppose that $\omega_{1}=3.17 \mathrm{rad} / \mathrm{s}, \omega_{2}=$ $7.38 \mathrm{rad} / \mathrm{s}$ with damping $\xi_{1}=0.001, \xi_{2}=0.015$, We suppose $F=\left[\begin{array}{ll}F_{1} & F_{2}\end{array}\right]$, where the coupling coefficients of the first two bending modes are $F_{1}=1.27814, F_{2}=$

$$
\left[\begin{array}{cccccc}
V_{1}^{\top}(A+B \omega K)^{\top}+(A+B \omega K) V_{1} \hat{P}-\kappa V_{1}+V_{1}^{\top}(A+B \omega K)^{\top} & B & V_{1}^{\top} C^{\top} & V_{1}^{\top} & V_{1}^{\top} K^{\top} \omega \\
* & \Xi_{22} & \kappa B & 0 & 0 & 0 \\
* & * & -\gamma^{2} I & 0 & 0 & 0 \\
* & * & * & -I & 0 & 0 \\
* & * & * & * & -Z^{-1} & 0 \\
* & * & * & * & * & -R^{-1}
\end{array}\right]<0
$$

From Schur Complement theory, we have

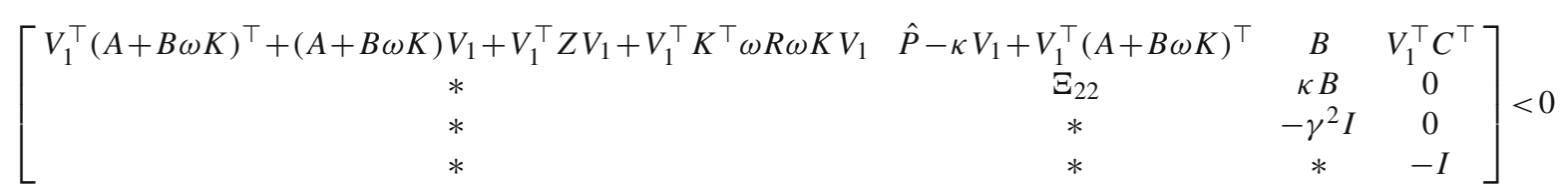

Then, pre-multiplying (25) by $\operatorname{diag}\left\{V_{1}^{-\top}, V_{1}^{-\top}\right.$, $I, I\}$ and post-multiplying by $\operatorname{diag}\left\{V_{1}^{-1}, V_{1}^{-1}, I, I\right\}$ and defining some matrices as follows:

$V_{1}^{-1}=\Gamma_{1}, \kappa V_{1}^{-1}=\Gamma_{2}, V_{1}^{-\top} \hat{P} V_{1}^{-1}=P$

Thus, (25) became to (12). From Theorem 1, it is clear that the closed-loop system (10) is asymptotically stable and satisfies $\|y(t)\|_{2}<\gamma\|w(t)\|_{2}$. The proof is completed.

Remark 3 By employing Lemma 1 and the fault description (7), the partial loss of actuator effectiveness problem is transformed into uncertain parameters problem. Thus, a feasible robust FTC scheme is obtained in
Table 1 The corresponding matrices $V_{1}$ and $W$ under different control effectiveness factors

Control effectiveness The corresponding matrices factor

\begin{tabular}{ll}
\hline$\hat{\omega}=1$ & $V_{1}=\left[\begin{array}{cc}0.1179 & -0.0105 \\
-0.0123 & 0.0022\end{array}\right]$, \\
$W=\left[\begin{array}{ll}0.0319 & -0.0302\end{array}\right]$ \\
$\hat{\omega}=0.5$ & $V_{1}=\left[\begin{array}{cc}0.1173 & -0.0104 \\
-0.0122 & 0.0022\end{array}\right]$, \\
& $W=\left[\begin{array}{cc}0.0639 & -0.0606\end{array}\right]$ \\
$\hat{\omega}=0.2$ & $V_{1}=\left[\begin{array}{cc}0.1046 & -0.0083 \\
-0.0095 & 0.0015\end{array}\right]$, \\
& $W=\left[\begin{array}{ll}0.1299 & -0.1162\end{array}\right]$
\end{tabular}


Table 2 The controller parameters under different control effectiveness factors

\begin{tabular}{lll}
\hline $\begin{array}{l}\text { Control effec- } \\
\text { tiveness factor }\end{array}$ & $\begin{array}{l}\text { Fault-tolerant } \\
\text { controller gain }\end{array}$ & $\begin{array}{l}\text { Upper bound of } \\
\text { performance index }\end{array}$ \\
\hline$\hat{\omega}=1$ & $K=[-2.3129-24.8248]$ & $J^{*}=11.7992$ \\
$\hat{\omega}=0.5$ & $K=[-4.6242-49.7914]$ & $J^{*}=11.8874$ \\
$\hat{\omega}=0.2$ & $K=[-11.0067-134.4086]$ & $J^{*}=11.0721$ \\
\hline
\end{tabular}

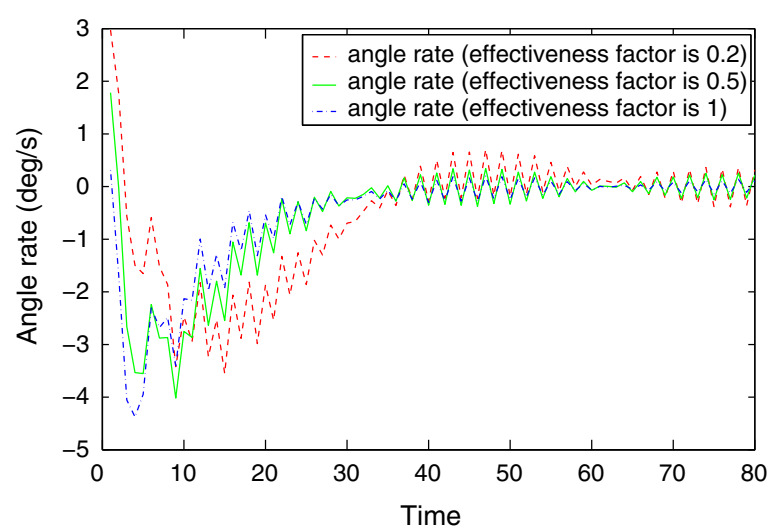

Fig. 1 The responses of pitch attitude angle and angle rate under partial actuator failure

Fig. 2 The responses of control effect when $\hat{\omega}=0.2, \hat{\omega}=0.5$, and $\hat{\omega}=1$

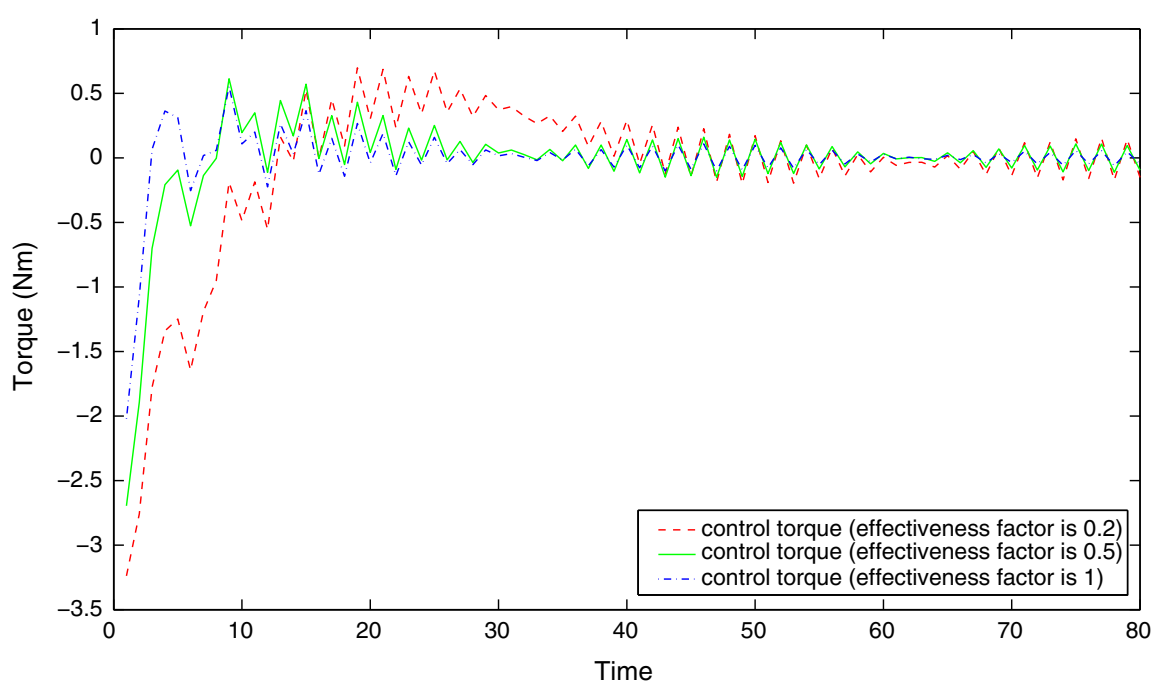

$0.91756, J=35.72 \mathrm{~kg} \mathrm{~m}^{2}$ is the nominal principal moment of inertia of pitch axis. The flexible spacecraft is supposed to move in a circular orbit with the altitude of $500 \mathrm{~km}$, then the orbit rate is $0.0011 \mathrm{rad} / \mathrm{s}$. The initial pitch attitude of the spacecraft are $\theta(0)=0.08 \mathrm{rad}$, $\dot{\theta}(0)=0.04 \mathrm{rad} / \mathrm{s}$. And $H_{\infty}$ performance index is supposed to $\gamma=2.5$ and the given matrices are chosen as $Z=\left[\begin{array}{llll}0.25 & 0.1 ; & 0.1 & 0.5\end{array}\right], \quad R=0.31, C=\left[\begin{array}{llll}1 & 0 ; & 0 & 1\end{array}\right]$.
The results we obtained by using Theorem 2 are given in Tables 1 and 2.

Figure 1 shows the responses of pitch attitude angle and angle rate under partial actuator failure, which the control effectiveness factors are chosen as when $\hat{\omega}=0.2, \hat{\omega}=0.5 \hat{\omega}=1$ respectively. Figure 2 shows the responses of control effect when $\hat{\omega}=0.2, \hat{\omega}=$ $0.5 \hat{\omega}=1$. From these figures, it is clear that the 
response performance can be guaranteed under the robust FT controller.

\section{Conclusion}

In this paper, a robust FTC scheme has been investigated for flexible spacecraft. The LMI-based conditions are formulated for the existence of the admissible controller, which ensures that the faulty closed-loop system is asymptotically stable with a $H_{\infty}$ disturbance attenuation level and partial loss of actuator effectiveness. Numerical simulations have shown the performance of the attitude control system. Further improvement in composite disturbance-observer with feedback control for flexible spacecraft will be considered in our future work.

Acknowledgments This work was supported in part by Major State Basic Research Development Program of China (973 Program) under Grant 2012CB720003, by the National Science Foundation of China under Grant Numbers 91016004 and 60904025, in part by Qing Lan project of Jiang Su province.

Open Access This article is distributed under the terms of the Creative Commons Attribution License which permits any use, distribution, and reproduction in any medium, provided the original author(s) and the source are credited.

\section{References}

1. Nagashio, T., Kida, T., Ohtani, T., Hamada, Y.: Design and implementation of robust symmetric attitude controller for ETS-VIII spacecraft. Control Eng. Pract. 18(12), 1440-1451 (2010)

2. Gennaro, S.D.: Adaptive robust tracking for flexible spacecraft in presence of disturbance. J. Optim. Theory Appl. 21, 545-568 (1998)

3. Yang, C.D., Sun, Y.P.: Mixed $H_{2} / H_{\infty}$ state-feedback design for microsatellite attitude control. Control Eng. Pract. 10, 951-970 (2002)

4. Hu, Q.: Variable structure maneuvering control with timevarying sliding surface and active vibration damping of flexible spacecraft with input saturation. Acta Astronaut. 64, 1085-1108 (2009)

5. Hu, Q., Ma, G.F.: Variable structure control and active vibration suppression of flexible spacecraft during attitude maneuver. Aerosp. Sci. Technol. 9(4), 307-317 (2005)

6. Dong, C., Xu, L., Chen, Y., Wang, Q.: Networked flexible spacecraft attitude maneuver based on adaptive fuzzy sliding mode control. Acta Astronaut. 65, 1561-1570 (2009)

7. Liu, H., Guo, L., Zhang, Y.: An anti-disturbance PD control scheme for attitude control and stabilization of flexible spacecraft. Nonlinear Dyn. 67(3), 2081-2088 (2012)

8. Hu, Q., Ma, G.F.: Control of three-axis stabilized flexible spacecraft using variable structure strategies subject to input nonlinearities. J. Vib. Control 12(6), 659-681 (2006)
9. Yu, X., Jiang, J.: Hybrid fault-tolerant flight control system design against partial actuator failures. IEEE Trans. Control Syst. Technol. 20(4), 871-885 (2012)

10. Karpenko, M., Sepehri, N.: Hardware-in-the-loop simulator for research on fault tolerant control of electro-hydraulic actuators in a flight control application. Mechatronics 19(7), 1067-1077 (2009)

11. Liu, H., Guo, L., Zhang, Y.: Composite attitude control for flexible spacecraft with simultaneous disturbance attenuation and rejection performance. Proc. Inst. Mech. Eng. 226, 154-161 (2012)

12. Benosman, M., Lum, K.Y.: Passive actuators fault-tolerant control for affine nonlinear systems. IEEE Trans. Control Syst. Technol. 18(1), 152-163 (2010)

13. Wang, R.R., Wang, J.M.: Passive actuator fault-tolerant control for a class of overactuated nonlinear systems and applications to electric vehicles. IEEE Trans. Veh. Technol. 62(3), 972-985 (2013)

14. Liu, M., Cao, X.B., Shi, P.: Fault estimation and tolerant control for fuzzy stochastic systems. IEEE Trans. Fuzzy Syst. 21(2), 221-229 (2013)

15. Jiang, B., Gao, Z.F., Shi, P., Xu, Y.F.: Adaptive fault-tolerant tracking control of near-space vehicle using Takagi Sugeno fuzzy models. IEEE Trans. Fuzzy Syst. 18(5), 1000-1007 (2010)

16. Shen, Q.K., Jiang, B., Cocquempot, V.: Fuzzy logic systembased adaptive fault-tolerant control for near-space vehicle attitude dynamics with actuator faults. IEEE Trans. Fuzzy Syst. 21(2), 289-300 (2013)

17. Zhang, Y.M., Jiang, J.: Bibliographical review on reconfigurable fault-tolerant control systems. IFAC Annu. Rev. Control 32(2), 229-252 (2008)

18. Ye, D., Yang, G.H.: Adaptive fault-tolerant tracking control against actuator faults with application to flight control. IEEE Trans. Control Syst. Technol. 14(6), 1088-1096 (2006)

19. Blanke, M., Kinnaert, M., Lunze, J., Staroswiecki, M.: Diagnosis and Fault Tolerant Control. Springer-Verlag, London (2006)

20. Liu, G.J., Wang, D.J., Li, Y.C.: Active fault tolerant control with actuation reconfiguration. IEEE Trans. Aerosp. Electron. Syst. 40(3), 1110-1117 (2004)

21. Qian, M.S., Jiang, B., Xu, D.Z.: Fault tolerant tracking control scheme for UAV using dynamic surface control technique. Circuits Syst. Signal Process. 31(5), 1713-1729 (2012)

22. Li, X.F., Guo, L., Zhang, Y.M.: A composite disturbance observer and $H_{\infty}$ control scheme for flexible spacecraft with time-varying input delay. In: 31th Chinese Control Conference, pp. 2824-2829. Hefei (2012)

23. Li, T., Guo, L., Sun, C.Y.: Robust stability for neural networks with time-varying delays and linear fractional uncertainties. Neurocomputing 71, 421-427 (2007)

24. Li, T., Guo, L., Lin, C.: Stability criteria with less LMI variables for neural networks with time-varying delay. IEEE Trans. Circuits Syst. II 55(11), 1188-1192 (2008)

25. Li, T., Yao, X.M., Wu, L.Y., Li, J.Q.: Improved delaydependent stability results of recurrent neural networks. Appl. Math. Comput. 19, 9983-9991 (2012) 